\title{
Isolation and Taxonomy of Filamentous Fungi in Endodontic Infections
}

\author{
Cinthya Gomes, DDS, MSc, PbD, * Sandra Fidel, DDS, MSc, PhD, ${ }^{\dagger}$ Rivail Fidel, DDS, MSc, PhD, ${ }^{\dagger}$ \\ and Maria Inês de Moura Sarquis, MSc, PbD ${ }^{\ddagger}$
}

\begin{abstract}
Introduction: The purpose of this study was to research the presence of filamentous fungi in the root canals of teeth with pulp necrosis and periapical lesions and to perform the taxonomic study of the isolates. Methods: Samples collected from 60 root canals with sterile absorbent paper points were inoculated in Sabouraud dextrose agar supplemented with chloramphenicol. The material was cultured from 7-14 days at room temperature to observe mycelial growth. Samples with growth were seeded in media specific for microculture (CYA agar, malt extract agar, or potato dextrose agar). Taxonomy was evaluated by macroscopic examination and optical microscopy. Results: Filamentous fungi were isolated in situ from 17 of 60 samples (28.3\%). Considering the positive root canal isolates, the genus Aspergillus was isolated from 7 samples (41\%); 4 species were identified: Aspergillus ustus, A. granulosus, $A$. niger, and $A$. sydowii. Emericella quadriluniata, sexual form of Aspergillus, was isolated from 1 sample. Penicillium species (Penicillium implicatum, P. micsynvisk, $P$. lividum, and $P$. citrionigrum) were isolated from 4 samples (24\%). Fusarium species (Fusarium moniliforme and F. melanochorum) were isolated from 2 samples (12\%). The species Aureobasidium pullulans, Exophiala jeanselmei, Eurotium amstelodame, and Cladosporium sphaerospermum were isolated from 1 sample each. Conclusions: The root canals of teeth with pulp necrosis and periapical lesions might present positive culture for filamentous fungi. (J Endod 2010;36:626-629)
\end{abstract}

\section{Key Words}

Filament fungi, necrotic pulp, periradicular disease, root canals, taxonomy
The presence of fungi in root canals was first reported by Grossman (1), who found fungal evidence in $17 \%$ of the evaluated samples. Fungi and yeasts have been isolated from root canals of teeth with pulp necrosis and apical periodontitis (2-4) and have been reported as a potential cause of endodontic failure in root-filled teeth (5-7). The presence of these pathogens has been attributed to opportunistic invasion in infected root canals (8).

During and after endodontic therapy of teeth with asymptomatic apical periodontitis, Saccharomyces cerevisiae was isolated from the patient's blood and root canal (9). Waltimo et al (10) isolated Geotrichum candidum, Candida albicans, C. guilliermimondii, C. glabrata, and C. inconspicua from root canals in pure cultures and together with bacteria.

Egan et al (2) evaluated the prevalence of yeasts in saliva and root canals of teeth associated with apical periodontitis and found that $C$. albicans and Rhodotorula mucilaginos $a$ were the most prevalent isolates.

According to Ferrari et al (3), Enterococcus, enteric bacteria, and fungi might be present in primary endodontic infections. The presence of fungi in the root canals of teeth with pulp necrosis and periapical lesion in human immunodeficiency virus (HIV) - and HIV+/acquired immune deficiency syndrome (AIDS) patients was evaluated in a recent study (11). Candida species were present on tooth surface in $37 \%$ of the HIV patients, and none was found in the root canals of these subjects. However, the Candida species present on tooth surface of HIV+/AIDS subjects were the same as those retrieved from their root canals.

C. albicans is the most commonly isolated yeast species from infected root canals and is potentially pathogenic $(4,7,12)$. However, there are no reports on the presence of filamentous fungi in endodontic infections, although these pathogens have already been isolated from infections in other body sites $(13,14)$. Like yeast, filamentous fungi might play a role in the pathogenesis of the periradicular diseases (4).

The main purpose of the present study was to investigate the presence of filamentous fungi in root canals of teeth with pulp necrosis and periradicular lesions by culture methods. In addition, a taxonomic study was performed in the species isolated.

\section{Material and Methods}

The research protocol was independently reviewed by the Ethics in Research Committee of the State University of Rio de Janeiro, and the study design was approved. Sixty patients (33 female and 27 male) were selected at the clinic of the School of Dentistry of the State University of Rio de Janeiro, Brazil. All subjects were aged 2370 years and were included after responding to a health questionnaire and signing an informed consent form authorizing collection and disclosure of their clinical data.

From the *Department of Endodontics, University Federal Fluminense, School of Dentistry, Nova Friburgo, RJ; ${ }^{\dagger}$ Department of Integrated Clinical Procedures, State University of Rio de Janeiro, Biomedical Centre, School of Dentistry, Rio de Janeiro, RJ; and `Department of Mycology, Laboratory of Fungi Culture Collection, Institute Oswaldo Cruz (FIOCRUZ), Rio de Janeiro, R.J, Brazil.

Address reprint requests to Prof. Dra. Cinthya Cristina Gomes, Presidente Backer Street, 116. Icaraí, 24220-041 Niterói, RJ, Brasil. E-mail address: cigomez@terra. com.br.

0099-2399/\$0 - see front matter

Copyright @ 2010 American Association of Endodontists.

doi:10.1016/j.joen.2010.01.016 
Cultures were performed from samples collected from 60 singlerooted and multi-rooted teeth that presented clinically and radiographically confirmed pulp necrosis and periradicular lesion. In single-rooted teeth, the main canal was sampled, whereas in the multi-rooted teeth only the largest canal in the root presenting the periapical radiolucency was sampled.

\section{Sample Collection}

After removal of the restoration and caries excavation, rubber dam isolation was performed, followed by antisepsis of the tooth and disinfection of the operative field with 5.25\% sodium hypochlorite. Neutralization was done with $5 \%$ sodium thiosulfate. Access to the pulp cavity was gained with a sterile spherical diamond bur at high speed, after which the operative field was cleaned with the same substances. The root canal was irrigated with sterile saline, and a sterile K-file of size compatible with the apical canal diameter and length, as established by a preoperative radiograph, was passively introduced. Root canal length was established with an apex locator and confirmed radiographically. A sterile Hedstrom file was used to remove dentin in the form of chips.

Three sterile absorbent paper points were used for material collection from each root canal. Within a field isolated by 2 alcohol lamps, each absorbent paper point was introduced into the root canal at $1 \mathrm{~mm}$ short of the root apex, kept for 1 minute, and transferred to a test tube containing Sabouraud agar (Difco Laboratories, Detroit, MI), with added $0.5 \%$ chloramphenicol (Medley, Campinas, SP, Brasil) to inhibit bacterial growth. An open Petri dish containing the same culture medium (Sabouraud agar with added $0.5 \%$ chloramphenicol) was placed within the field isolated by 2 alcohol lamps to ascertain isolation accuracy (negative control). The positive control consisted of a Petri dish containing the same culture medium that remained open and out of the isolated field during sample collection to verify whether environmental fungi grew and establish whether they coincided with the canal isolates. The Petri dishes and test tubes containing the collected material were kept at room temperature for periods of 7-14 days. Mycelial growth was checked every 24 hours. The tubes and dishes that presented fungal colony growth were sent to the Institute Oswaldo Cruz, Laboratory of Fungi Culture Collection for colony macroscopic and microscopic taxonomic identification and classification according to the classic mycologic techniques described in the literature specific for each taxon.

\section{Direct Fungal Colony Examination}

A colony fragment was retrieved from a monosporic culture, placed on a glass slide, stained with Amann lactophenol cotton blue (Difco Laboratories) cytoplasmic dye and covered with a coverslip. This technique was used to prepare the material for examination with an optical microscope to visualize the vegetative and reproductive fungal propagation of mycelium. The glass slides hereby prepared were designated as temporary because the observed structures were fragmented and allowed identifying only phylum and the possible genus, not the species.

\section{Sampling From Specific Culture Media}

A specific culture medium (malt extract agar, Czapeck yeast extract agar [CYA agar], or potato dextrose agar; Difco Laboratories) was used for each fungus, depending on its characteristics as determined by direct examination. A colony fragment was collected by using a sterile platinum loop and inoculated onto the specific culture medium (inoculum point) to favor fungal growth and sporulation. The colonies were incubated at room temperature and observed every 24 hours, because each fungal strain has a different growth time according to its taxonomic group. The colonies were then examined macroscopically.

\section{Macroscopic Identification}

The identification and authentication of the fungal species were performed by analysis of the macroscopic characteristics related to each strain: colony texture, color of the front and reverse side, pigment production, colony size, growth time, exudate production or not. All characteristics were recorded and evaluated for being potentially important for fungal line classification.

\section{Microscopic Identification}

Mycelial fragments of each isolate were carefully examined in the preparations obtained from the microcultures inoculated onto specific culture media as well as glass slides and coverslips, according to the technique of Rivalier and Seydel (15). This technique is performed by using a sterile $15-\mathrm{cm}$ diameter Petri dish whose bottom is covered with filter paper or cotton mesh, and 2 glass slides and 2 coverslips are placed onto a U-shaped glass rod. The assembly was wrapped in Kraft paper and sterilized in Pasteur oven at $37^{\circ} \mathrm{C}$ during 2 hours. Adequate culture media for each fungal genus or species were prepared and poured in the Petri dishes. After solidification, the media were cut with a sterile scalpel blade into $1-\mathrm{cm}^{2}$ squares, which were placed on each microculture chamber slide by using a sterile platinum loop. Each fungal colony was inoculated onto the 4 sides of each culture medium square, and a coverslip was placed. Filter paper soaked with sterile distilled water was placed inside the chamber to maintain humidity. After labeling, each dish was maintained in the microculture chamber at room temperature for 7 days or more, depending on the fungus, for colony growth and sporulation observation. After adequate growth time, glass slides and coverslips were assembled separately. Lactophenol cotton blue was dripped onto the glass slides. Coverslips containing vegetative and reproductive mycelia were retrieved with sterile tweezers and placed on the stained glass slides. The culture medium squares with the grown fungi were fixed in formalin for 24 hours; the glass slides were examined with an optical microscope for taxonomic classification of the species. Taxonomic identification was performed both macroscopically and microscopically (16-24).

\section{Results}

Seventeen $(28.3 \%)$ of 60 collected samples presented positive culture for filamentous fungi (Table 1).

Considering the positive root canal isolates, the genus Aspergillus (Fig. $1 A, B$ ) was isolated in situ from 7 samples (41\%). The fungal strain Aspergillus ustus (Fig. 1C) was isolated from 3 samples. The species A. granulosus, A. niger, A. sydowii, and Emericella quadriluniata were isolated from 1 sample each.

The genus Penicillium was isolated in situ from 4 samples (24\%); the following species were identified: Penicillium implicatum, P. micsynvisk, P. lividum, and P. citrionigrum. The genus Fusarium was

TABLE 1. Frequency distribution of the types of filamentous fungi isolated from root canal samples

\begin{tabular}{lc}
\hline Type of fungus & Number of samples (\%) \\
\hline Aspergillus & $7(41)$ \\
Penicillium & $4(24)$ \\
Fusarium & $2(12)$ \\
Aureobasidium & $1(6)$ \\
Exophiala & $1(6)$ \\
Eurotium & $1(6)$ \\
Cladosporium & $1(6)$ \\
\hline
\end{tabular}




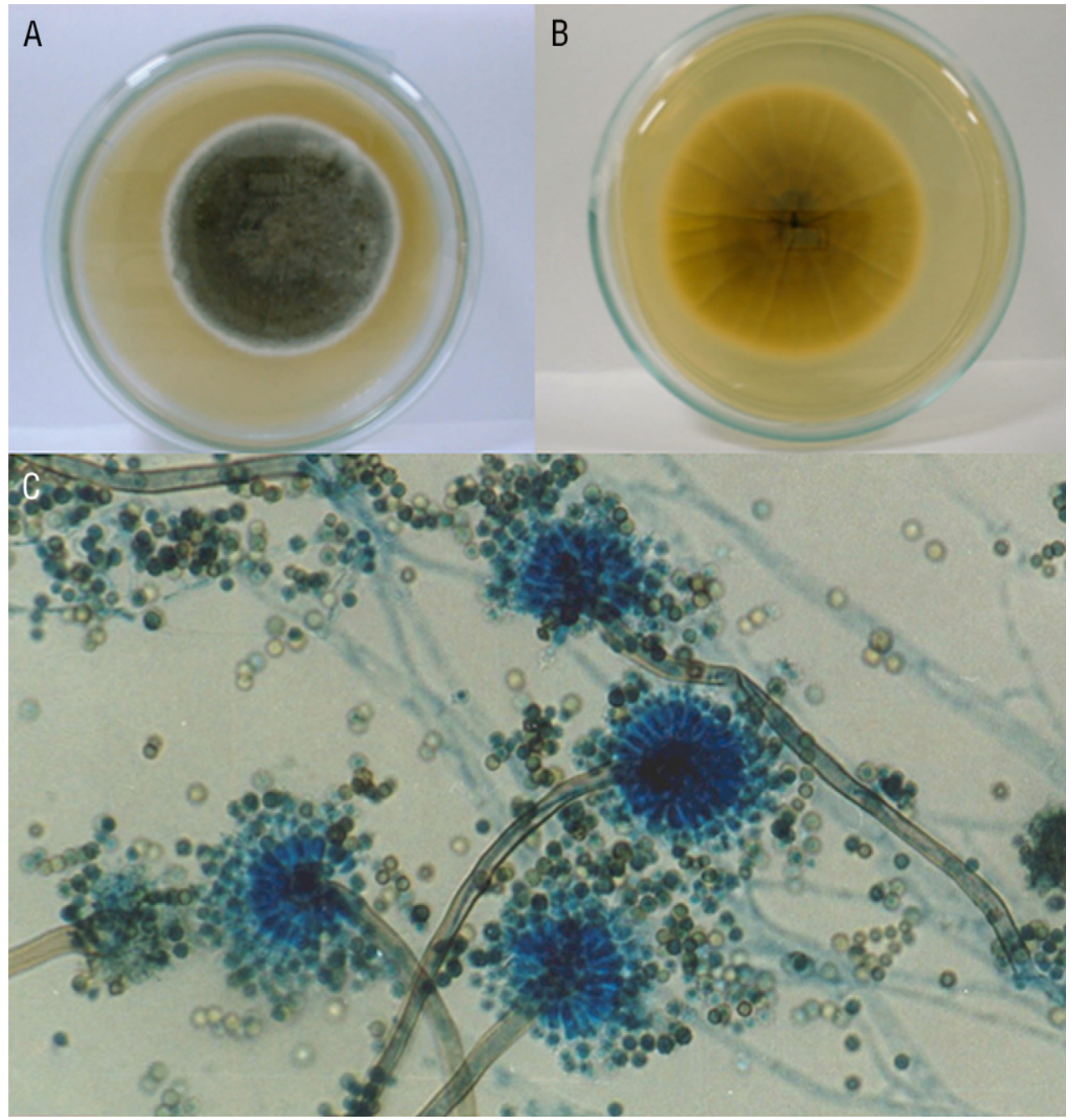

Figure 1. Aspergillus seeded in malt extract agar at $37^{\circ} \mathrm{C}$. (A) Front and $(B)$ reverse side of the Petri dish. $(C)$ Optical microscopic image of $A$. ustus (original magnification, $150 \times)$.

isolated from 2 samples (12\%); the following species were identified: Fusarium moniliforme and $F$. melanochorum.

The species Aureobasidium pullulans, Exophiala jeanselmei, Eurotium amstelodame, and Cladosporium sphaerospermum were isolated from 1 sample each.

There was no mycelial growth in the negative control (Petri dishes placed in the field isolated by the 2 alcohol lamps). However, there was growth of fungi different from the root canal isolates in the positive control (Petri dishes left open out of the isolated field to assess environmental contamination). In addition, no pure cultures were observed.

\section{Discussion}

It is well-established that periradicular diseases are caused by the interplay of different microorganism species $(5,10,12,25,26)$, which characterize a mixed root canal space infection. Furthermore, previous studies have demonstrated the presence of fungi in teeth with primary and secondary endodontic infection (10, 25-27). Taking into consideration the whole human organism, the most commonly encountered species of opportunistic fungal pathogens are members of the genera Candida and Aspergillus (4). Inside the context of the periradicular diseases, $C$. albicans represents the most commonly isolated yeast species from infected root canals $(4,7,12)$. However, although filamentous fungi have already been isolated in other body sites, including infections in the maxillary sinus $(13,14)$, to the best of our knowledge there is no evidence demonstrating their presence in the root canal space thus far. Thus, the present study is the first report showing the presence of filamentous fungi in infected root canals. Previously, a scanning electron microscopic study identified structures compatible with filamentous fungi measuring $3-5 \mu \mathrm{m}$ in diameter with distinct cell wall and dense nucleus (25). However, the authors were unable to identify the species, emphasizing that the knowledge of oral mycobiota is limited by the lack of taxonomists, and as a consequence, several isolated species remain unidentified. Sen et al (26) have also reported the presence of structures compatible with filamentous fungi measuring 4-6 $\mu \mathrm{m}$ in diameter, but they have not biomorphologically identified the species as well. In the present study, all isolated species have diameters compatible with those reported by Nair et al (25) and Sen et al. Therefore, the microorganisms isolated in both above-mentioned studies $(25,26)$ could be filamentous fungi that were not taxonomically identified.

Fungi possess virulence attributes including adaptability to a variety of environmental conditions, adhesion to a variety of surfaces, production of hydrolytic enzymes, morphologic transition, biofilm formation, 
and evasion and immunomodulation of host defense, which might play a role in periradicular disease pathogenesis (4). The filamentous fungi isolates in the present study (A. niger, Aureobasidium pullulans, Cladosporium sphaerospermum, Exophiala jeanselmei) are able to produce melanin; this hydrophobic pigment is located on conidium surfaces and is recognized as virulence factor, because it has protective ability against damages caused by macrophages and neutrophils on the pathogen (28).

In conclusion, the present study reinforces fungi presence in endodontic infections because filamentous fungi were isolated straight from root canals of teeth with pulp necrosis and periradicular lesions. Further studies should be developed to confirm the present findings, preferably by using molecular methods for microbiologic identification. Thus, the full role of the fungi in periradicular disease pathogenesis can be better elucidated in the near future.

\section{References}

1. Grossman LI. Root canal therapy. 3rd ed. London: Henry Kimpton; 1952.

2. Egan MW, Spratt DA, Ng YL, Lam JM, Moles DR, Gulabivala K. Prevalence of yeasts in saliva and root canals of teeth associated with apical periodontitis. Int Endod J 2002; 35:321-9.

3. Ferrari PHP, Cai S, Bombana AC. Effect of endodontic procedures on enterococci, enteric bacteria and yeasts in primary endodontic infections. Int Endod J 2005;38: 372-80.

4. Siqueira JF Jr, Sen BH. Fungi in endodontics infection. Oral Surg Oral Med Oral Pathol Oral Radiol Endod 2004;97:632-41.

5. Pinheiro ET, Gomes BP, Ferraz CC, Souza EL, Teixeira FB, Souza-Filho FJ. Microorganisms from canals of root-filled teeth with periapical lesions. Int Endod J 2003; 36:1-11.

6. Siqueira JF Jr, Roças IN. Polymerase chain reaction-based analysis of microorganisms associated with failed endodontic treatment. Oral Surg Oral Med Oral Pathol Oral Radiol Endod 2004;97:85-94

7. Waltimo TMT, Sen BH, Meurman JH, Orstavik D, Haapasalo MPP. Yeasts in apical periodontitis. Crit Rev Oral Biol Med 2003;14:128-37.

8. Nair PN, Sjogren U, Krey G, Kahnberg KE, Sundqvist G. Light and electron microscopic studies of root canal flora and periapical lesions. J Endod 1987;13:29-39.

9. Debelian GJ, Olsen I, Tronstad L. Bacteremia in conjunction with endodontic therapy. Endod Dent Traumatol 1995;11:142-6.
10. Waltimo TMT, Sirén EK, Torkko HL, Olsen I, Haapasalo MP. Fungi in therapyresistant in apical periodintitis. Int Endod J 1997;30:96-101.

11. Chugal N, Fleischmann J, Sondej M, Spängberg L. Quantitative isolation of fungi from root canals: a methodological study. IADR/AADR/CADR General Session and Exhibition Online, New Orleans, LA. Available at: http://iadr.confex.com/iadr/ 2007orleans/techprogram/abstract_91685. Accessed March 8, 2008.

12. Sunde PT, Olsen I, Debelian GJ, Tronstand L. Microbiota of periapical lesions refractory to endodontic therapy. J Endod 2002;28:304-10.

13. Legent F, Billet J, Beauvillain C, Bonnet J, Miegeville M. The role of dental canal fillings in the development of Aspergillus sinusitis: a report of 85 cases. Arch Otorhinolaryngol 1989;246:318-20.

14. Mensi M, Piccioni M, Marsili F, Nicolai P, Sapelli CL, Latronico N. Risk of maxillary fungus ball in patients with endodontic treatment on maxillary teeth: a case-control study. Oral Surg Oral Med Oral Pathol Oral Radiol Endod 2007;103:433-6.

15. Rivalier E, Seydel S. Nouveau procedé de culture sur lames gélosées applique a l'étude microscopique des champignons des teignes. Ann Parasitol 1932;10:444-52.

16. Garro J, Gene J. Fusarium infections criteria for the identification on the responsible species. Mycoses 1992;35:109-14.

17. Gerlach W, Nirenberg $H$. The genus Fusarium: a pictorial atlas. Berlin, Germany: Institute fur Mikrobiologie Press, 1982:406.

18. Hawksworth DL, Sutton BC, Ainsworth GD. Dictionary of fungi 7th ed. Kew, Surrey, England: CAB Press, 1983:445.

19. Hawksworth DL, Kirk PM, Sutton BC, Pegler DN. Dictionary of fungi 8th ed. Wallinglord, UK: CAB International, 1995:816.

20. Pitt JI. The genus Penicillium. Australia: Academic Press, 1979:635.

21. Pitt JI. A laboratory guide to common Penicillium species. Australia: Academic Press, 1985:182.

22. Raper KB, Fennel DI. The genus Aspergillus, 690. Baltimore, MD: Williams \& Wilkins Co; 1965.

23. Samson RA, Stolk AC, Hadlok R. Revision of the subsection fasciculate of Penicillium and some allied species. Studies in Mycology 1976;11.

24. Samson RA. A compilation of the Aspergilli described since 1965. Studies in Mycology 1979;18:37-8.

25. Nair PNR, Sjögren U, Krey G, Kahnberg K, Sundqvist G. Intraradicular bacteria and fungi in root-filled, asymptomatic human teeth with therapy-resistant periapical lesions: a long-term light and electron microscopic follow-up study. J Endod 1990;16:580-8.

26. Sen BH, Piskin B, Demirci T. Observation of bacteria and fungi in infected root canals and dentinal tubules by SEM. Endod Dent Traumatol 1995;11:6-9.

27. Siqueira JF Jr. Endodontics infections: concepts, paradigms, and perspectives. Oral Surg Oral Med Oral Pathol 2002;94:281-93.

28. Langfelder K, Streibel M, Jahn B, Haase G, Brakhage A. Biosynthesis of fungal melanins and their importance for pathogenic fungi. Fungal Genet Biol 2003;38:143-58. 